\title{
Blind Image Deconvolution Using Machine Learning for Three-Dimensional Microscopy
}

\author{
Tal Kenig, Zvi Kam, and Arie Feuer, Fellow, IEEE
}

\begin{abstract}
In this work, we propose a novel method for the regularization of blind deconvolution algorithms. The proposed method employs example-based machine learning techniques for modeling the space of point spread functions. During an iterative blind deconvolution process, a prior term attracts the point spread function estimates to the learned point spread function space. We demonstrate the usage of this regularizer within a Bayesian blind deconvolution framework and also integrate into the latter a method for noise reduction, thus creating a complete blind deconvolution method. The application of the proposed algorithm is demonstrated on synthetic and real-world three-dimensional images acquired by a wide-field fluorescence microscope, where the need for blind deconvolution algorithms is indispensable, yielding excellent results.
\end{abstract}

Index Terms-Blind deconvolution, deblurring, machine learning, PCA, kernel PCA, microscopy.

\section{INTRODUCTION}

A $\mathrm{N}$ image acquired by an imaging system may be Adegraded due to numerous reasons. Two of the most common reasons for image degradation are blur and statistical noise. Sometimes the blurring operation can be assumed to be linear and shift invariant and therefore the acquired image can be modeled as a convolution of the imaged object with some convolution kernel representing the imaging system. This convolution kernel is often referred to as the point spread function (PSF) of the imaging system. Neglecting the effect of statistical noise, the blurring is mathematically modeled as a convolution and therefore the process of inverting this operation is termed deconvolution. In addition, there are situations where explicit knowledge of the convolution kernel cannot be assumed. Therefore, inverting the convolution operation under those circumstances is termed blind deconvolution (BD).

In general, the assumed image degradation model can be formulated as

$$
g=n(h * f)
$$

and in the discrete case, we formulate (1) explicitly as:

$$
g(\mathbf{x})=n\left(\sum_{\mathbf{k} \in \Omega} f(\mathbf{k}) h(\mathbf{x}-\mathbf{k})\right),
$$

- T. Kenig and A. Feuer are with the Electrical Engineering Faculty, Technion-Israel Institute of Technology, Haifa, Israel.

E-mail: talkenig@tx.technion.ac.il; feuer@ee.technion.ac.il.

- Z. Kam is with the Department of Molecular Cell Biology, Weizmann Institute of Science, Rehovot 76100, Israel.

E-mail:zvi.kam@weizmann.ac.il.

Manuscript received 7 May 2009; revised 12 Aug. 2009; accepted 3 Nov. 2009; published online 25 Feb. 2010.

Recommended for acceptance by $M$. Figueiredo.

For information on obtaining reprints of this article, please send e-mail to: tpami@computer.org, and reference IEEECS Log Number

TPAMI-2009-05-0287.

Digital Object Identifier no. 10.1109/TPAMI.2010.45. where:

- $\quad p$ is the dimension of the image. In this work, we refer to $p=3$,

- $\Omega \subset \mathbb{R}^{p}$ is the support in the object domain imaged by the imaging system,

- $\mathbf{x} \in \Omega$ is a $p$-tuple of discrete spatial coordinates corresponding to the spatial locations sampled by the imaging system,

- $f: \Omega \rightarrow \mathbb{R}$ denotes the imaged object,

- $h: \Omega \rightarrow \mathbb{R}$ denotes the imaging system PSF,

- $g$ denotes the acquired image,

- $n$ denotes a pixelwise noise function,

- $*$ denotes the $p$-dimensional convolution operator.

The goal of BD algorithms is to estimate $f$ and $h$ from $g$ and any additional prior knowledge available.

In what follows, we assume that the dominant source of statistical noise in the imaging system is photon noise. Photon noise is common to many imaging systems, as it originates from the stochastic nature of the photon detection process at the sensor and is distributed according to Poisson distribution [5], with its parameter being the noise-free gray level value at each pixel location. The photon noise can be characterized as follows:

$$
p(g(\mathbf{x}) \mid f, h)=\frac{(f * h)(\mathbf{x})^{g(\mathbf{x})} e^{-(f * h)(\mathbf{x})}}{g(\mathbf{x}) !} .
$$

It can be easily observed that this type of noise is highly correlated with the signal, which makes it much more difficult to handle than additive noise.

It is not difficult to realize that problem (1) is ill-posed, in the sense that numerous solutions exist. This can be easily shown even without examining any specific method for solution. First, we note that the trivial solution $f \equiv g, h \equiv \delta$, where $\delta$ denotes the three-dimensional Dirac function, always exists. Second, if we examine (1), even without noise, from an information quantity point of view we can see that we have one image $g$ as input, from which we are required to estimate both $f$ and $h$. Hence, the problem at hand is 
underdetermined, i.e., there are more unknowns than inputs. Due to this ill-posedness, all BD methods use some sort of regularization. Many algorithms regularize the object of interest, usually by applying some sort of smoothness constraint see, e.g., [18], [30], [34], [36]. However, in general, the object we wish to reconstruct is unknown and regularizing it may have an adverse effect, especially in cases where it is known to have high frequency structures.

Contrary to that, the PSF we seek is usually directly related to the imaging system, thus providing us with some prior knowledge of its form. We note that in BD problems, although the PSF is not exactly known, it typically belongs to some distinct class. Therefore, we wish to integrate into the $\mathrm{BD}$ process as much accurate and specific information about the PSF as possible. Sometimes this information is theoretically available, since we know the general form of the PSF, e.g., in the cases of linear motion blur, atmospheric turbulence blur, and out-of-focus blur [6]. Even when this is not the case, there are many situations where the PSF can be experimentally measured under varying acquisition conditions, thus providing us a sampling of the class of PSFs.

We propose regularizing the PSF by attracting it to a space of admissible PSFs, denoted as PSF-space, known to represent the imaging system at hand. This is done by first performing example-based learning of the PSF-space by using linear principal component analysis (PCA) [49] or kernel PCA (KPCA) [57]. We then introduce into the deconvolution algorithm a term that attracts the PSF toward PSF-space.

This approach has never been proposed in the context of blind deconvolution, to the best of our knowledge. The inspiration for the proposed approach stems from the prolific activity in the field of statistical shape priors for segmentation using active contours in the past decade [13], [19], [20], [38], [54], [64].

The algorithm presented in this work has been developed for the specific application of restoring three-dimensional (3D) microscopic imagery. However, we present it in a general enough form to facilitate the application of the proposed algorithm for other uses of BD, as well.

We now outline the contents of this paper. In Section 2, we briefly review related work in the field of BD. Section 3 contains the main results of the Bayesian BD formulation used as a framework for the proposed method. Section 4 contains the main contribution of our work, which is a novel PSF regularization method, utilizing learning-based algorithms. Section 5 briefly discusses the convergence of the proposed algorithm. In Section 6, we provide the necessary details about wide-field fluorescence microscopic imaging, which is required for the understanding of the numerical experiments depicted in Section 7, which are conducted in order to validate the proposed algorithm. Finally, we conclude our work and suggest some further research topics in Section 8.

\section{Previous Work}

In general, BD algorithms can be divided into two categories: a priori blur identification methods and joint blur identification and image restoration methods [6]. The first category consists of algorithms which separate BD into two stages. The first stage consists of identification of the PSF. Once the PSF estimate is available, a nonblind deconvolution is carried out.
The earliest methods in this field are [7], [61], which assume that the PSF is of known parametric form and is completely characterized by its frequency domain zeros, which is frequently not the case. Another popular BD method in this field is zero sheet separation [37], in which the PSF is identified by separating the two-dimensional (2D) Z-transform of the corrupted image into two convolutive factors. In [56], the authors suggest calculating several restorations of the acquired images, using different PSFs. Those PSFs are obtained by acquiring, in advance, small, point-like objects under different imaging conditions. Then, the best image is selected either by some numerical measure or by visual inspection. Two other related methods for blur identification include generalized cross validation (GCV) [52] and kurtosis minimization [40]. Both methods assume an underlying parametric model for the PSF, perform multiple deconvolutions with different PSF parameters, and choose the PSF which leads to minimization of the GCV prediction error in [52] or the kurtosis of the restored image in [40]. Due to the need for performing multiple restorations, the deconvolution methods reported in [56], [52], and [40] are limited to computationally inexpensive algorithms. The authors of [56] use a fast, semi-3D deconvolution algorithm, despite the fact their problem is fully 3D, and the authors of [52] and [40] use linear, Wiener-like inverse filters for image restoration. Another recent method in the class of a priori blur identification methods which has been used in the context of microscopy relies on the assumption that the imaged object contains small point-like structures [16]. The latter are identified, cropped from the acquired image, and serve as a PSF estimate.

Most recent work in the field of BD is related to the class of joint image restoration and blur identification methods. The methods in this class are numerous and diverse, and we do not intend to cover them all. For a thorough review of $\mathrm{BD}$ methods the reader is referred to [6], [8], [34] and the references therein. In essence, the methods in this class can be categorized according to the deconvolution framework itself and the applied regularization.

One of the most popular BD frameworks is Bayesian BD, which is also used in our work. In Bayesian framework, the observed image and sometimes also the object and PSF are considered to be a single realization of a stochastic process. Some underlying probability distribution of those stochastic processes is assumed and a cost functional which aims at optimizing some statistical quantity is formulated and optimized. Usually, in creating this cost functional, one of the versions of the famous Bayes rule is utilized. Especially popular Bayesian methods are the maximum likelihood and maximum a posteriori methods, which are discussed in some detail in Section 3. Examples for the use of such methods can be found in [18], [22], [29], [30], [33], [65], [68] to name a few.

Due to the ill-posed nature of the BD problem, essentially all BD algorithms utilize prior knowledge in the deconvolution process, which is required in order to reduce the degrees of freedom of the problem. One class of regularization methods is the technique of projection onto convex sets (POCS). In this technique, some knowledge of the object or the PSF is forced, by projecting the intermediate object and/or 
PSF estimates onto some convex sets. For example, popular constraints used for POCS regularization are nonnegativity, spatial, and frequency domain supports, and phase information of the object. POCS methods can be used as regularizers in conjunction with other iterative methods, as in [1], [33], [34], [65], or as a BD method of itself as reported in [46], [66].

Another form of regularization used in BD is parametrization of the PSF. In these methods the PSF is either of known parametric form, or is assumed to be well approximated by a simple function dependent on a few parameters. Then, during the reconstruction process, only the PSF parameters need to be estimated, thus dramatically reducing the degrees of freedom in the problem. Examples for methods that use PSF parametrization are available in [43], [48].

Some regularization methods used for BD induce smoothness of the solution. Those methods usually incorporate into the BD process a term that attempts to minimize some differential quantity of the estimated object and/or PSF, such as the Laplacian, or an anisotropic regularization term, such as total variation [55]. Examples for this type of regularization in $\mathrm{BD}$ have been previously reported in [30], [67], and in [18] in the context of nonblind deconvolution.

The adaptive image deconvolution algorithm (AIDA) [30] contains a PSF regularization term which attracts the PSF estimate toward a given PSF. This approach can be viewed as a particular case of the approach we propose, where the PSF-space is degenerate and "learned" by a single example. This algorithm has been tested on 2D and 3D data sets, including 3D microscopy imagery, and we use it for comparison purposes in the sequel.

Another class of methods for regularization of BD algorithms are learning based algorithms. Not much research has been conducted in this field, and we note the recent work in [45], [47], [39]. Similar methods are also used for example-based super-resolution algorithms [23], [15]. In those works, the basic idea is that the prior knowledge required for solving the restoration problem can be learned from training data, i.e., a set of prototype images representing the same class of images as the ones processed. The training images are first corrupted in a similar manner to the expected degradation, thus creating image pairs. Then, the training images are cropped into small patches. During the reconstruction process, the surrounding patch of each image pixel is extracted, and similar degraded patches are found within the training data. The high quality patches paired with the found degraded patches are then used within the restoration process. While all of those methods share a similar idea, they differ in the way in which the training data is preprocessed, organized, and used.

We mention these methods since they are somewhat related to the method we propose, in the sense that they use example-based learning. However, we stress that this approach is fundamentally different from the one we propose in this paper. First, we do not make any assumption regarding the class of objects, whereas the above mentioned methods use very strong assumptions regarding the images to be processed. Second, we use machine learning methods in order to learn the parametrization of the PSF-space, and the objects which the learning process is employed upon are whole PSFs, rather than small image patches.

Finally, we note that much attention has been given to the problem of blind deblurring in the graphics and computer vision literature in recent years. Most of this activity focuses on the important problem of removing motion blur from natural images, see, e.g., [68], [31], [21], [32], [58], [50], [11]. In this context, we mention that our proposed method does not pertain to solving the problem of motion blur. This limitation stems from the learning methods we employ, which are incapable of capturing the properties of the motion blur PSF, since it is usually very diverse and unpredictable. In addition, some of this work addresses spatially variant blur [50], [11], involves acquisition of more than one photograph in special settings [68], or using dedicated acquisition devices [50], all of which are outside the scope of this work.

\section{BD FRAMEWORK}

The algorithm we propose in this work is based on the Maximum Likelihood Expectation Maximization (MLEM) and maximum a posteriori (MAP) algorithms. The MLEM algorithm was first introduced in the context of positron emission tomography reconstruction [59] and is similar to the Richardson-Lucy (RL) algorithm [41], [53]. ${ }^{1}$

\subsection{Maximum a Posteriori}

Maximum a posteriori is an algorithm which optimizes the following cost function:

$$
\hat{f}, \hat{h}=\arg \max _{f, h}\{p(f, h \mid g)\} .
$$

According to the Bayes rule, $p(f, h \mid g)=\frac{p(g \mid f, h) \cdot p(f, h)}{p(g)}$. We can assume with high confidence that $f$ and $h$ are statistically independent, so we get $p(f, h \mid g)=\frac{p(g \mid f, h) \cdot p(f) p(h)}{p(g)}$. The log likelihood of this expression is

$$
\begin{aligned}
& \log (p(f, h \mid g))=\log (p(g \mid f, h)) \\
& \quad+\log (p(f))+\log (p(h))-\log (p(g)) .
\end{aligned}
$$

We note that $\log (p(g))$ is not dependent upon $f$ or $h$. The term $\log (p(g \mid f, h))$ is governed by Poisson statistics, according to (2). The terms $\log (p(f))$ and $\log (p(h))$ can be interpreted as prior information regarding the object $f$ and the PSF $h$, respectively. At this point, we assume uniform distributions for $f$ and $h$, thus dropping the prior terms, which reduces the algorithm to the MLEM or RL. However, we will show in Sections 3.2 and 4 how we regularize the object and PSF estimates, respectively. By substituting (2) into (3) and differentiating, we arrive at the following iterative scheme:

$$
\begin{aligned}
& \hat{f}_{k+1}=\hat{f}_{k} \cdot\left[\left(\frac{g}{\hat{f}_{k} * \hat{h}_{k}}\right) * \hat{h}_{k}^{s}\right], \\
& \hat{h}_{k+1}=\frac{\hat{h}_{k}}{\sum_{\mathbf{x} \in \mathbf{\Omega}} \hat{f}_{k}(\mathbf{x})}\left[\left(\frac{g}{\hat{f}_{k} * \hat{h}_{k}}\right) * \hat{f}_{k}^{s}\right],
\end{aligned}
$$

1. The derivations of MLEM and RL stem from different assumptions, yet result in the same iterative process. See [8] for further details. 
where the superscript $s$ denotes symmetrical reflection: $\xi^{s}(\mathbf{x})=\xi(-\mathbf{x})$ and $k$ is the iteration index. Detailed derivation of the MLEM formulation can be found in [26], [8].

\subsection{MLEM with Integrated Denoising}

We neither posses any specific knowledge of our object $f$ nor wish to impose any general prior which assumes smoothness of any kind, since it may be inadequate for certain objects. However, it is implied by (3) that some regularization of the object estimate is required.

In order to overcome the noise, we use a generalization of a scheme from the field of astronomical imaging, of incorporating denoising within the MLEM framework [60]. This method is based on the observation that at each MLEM iteration $k$, the acquired image can be decomposed into two components: the estimated image at iteration $k$ and a residual: $g=\hat{f}_{k} * \hat{h}_{k}+R_{k}$. It can be noticed that the first term, $\hat{f}_{k} * \hat{h}_{k}$, is a smooth term (since $\hat{h}_{k}$ is a blurring kernel and as such is low-pass in nature), and therefore, the residual $R_{k}$ must contain the noise. This method suggests denoising $R_{k}$ at each iteration: $\bar{R}_{k}=\operatorname{Denoise}\left(R_{k}\right)$.

The authors of the original work [60] use wavelet-based denoising for the function Denoise(). However, in general there is no restriction regarding the denoising function. Using the aforementioned denoising scheme, the residualdenoised MLEM (RD-MLEM) iteration becomes:

$$
\begin{aligned}
R_{k} & =g-\hat{f}_{k} * \hat{h}_{k}, \\
\bar{R}_{k} & =\operatorname{Denoise}\left(R_{k}\right), \\
\hat{f}_{k+1} & =\hat{f}_{k} \cdot\left[\left(\frac{\hat{f}_{k} * \hat{h}_{k}+\bar{R}_{k}}{\hat{f}_{k} * \hat{h}_{k}}\right) * \hat{h}_{k}^{s}\right], \\
\hat{h}_{k+1} & =\frac{\hat{h}_{k}}{\sum_{\mathbf{x} \in \boldsymbol{\Omega}} \hat{f}_{k}(\mathbf{x})} \cdot\left[\left(\frac{\hat{f}_{k} * \hat{h}_{k}+\bar{R}_{k}}{\hat{f}_{k} * \hat{h}_{k}}\right) * \hat{f}_{k}^{s}\right] .
\end{aligned}
$$

In our experiments, the RD-MLEM was implemented using a $3 \times 3 \times 3$ median filter [28] for the Denoise() function, yielding satisfactory results.

We would like to briefly discuss our choice of the median filter. First, we note that the use of a linear filter is not desirable for this denoising task. If we denote by $\bar{g}_{k}=$ $\hat{f}_{k} * \hat{h}_{k}+\bar{R}_{k}$ the denoised estimate of $g$ at iteration $k$ and assume that Denoise() is a linear shift-invariant filter characterized by the convolution kernel $m$, we get

$\bar{g}_{k}=\hat{f}_{k} * \hat{h}_{k}+m *\left(g-\hat{f}_{k} * \hat{h}_{k}\right)=(\delta-m) *\left(\hat{f}_{k} * \hat{h}_{k}\right)+m * g$.

Since $\hat{f}_{k} * \hat{h}_{k}$ is a smooth term and $\delta-m$ is a high-pass filter, ${ }^{2}$ we expect the term $(\delta-m) *\left(\hat{f}_{k} * \hat{h}_{k}\right)$ to be negligible. This means that $\bar{g}_{k}$ is essentially a low-pass filtered version of $g$. Thus, we further blur the image $g$, which is in contrast to the goal of the deconvolution process. Another important detail is that we denoise $g-\hat{f}_{k} * \hat{h}_{k}$, which ideally contains only noise, and in practice, we do not expect to be of any specific structure. Therefore, we refrain from using complicated denoising methods that assume the presence of edges or any other kind of structure in the denoised image. We choose to use the median filter, which is nonlinear,

2. Since $m$ is a low-pass filter. efficient, and does not make any assumption regarding the underlying structure of the denoised image.

Finally, we note that although this scheme for noise reduction is very simple, in practice we found it to be very efficient in suppressing noise, without compromising fine detail in the images. This property of the denoising scheme stems from the fact it does not impose any constraints regarding the restored object, but instead, heavily relies on the convolutive imaging model.

\section{PSF Regularization by EXAMPLe-BASEd LEARNING}

In this section, we introduce the main novelty of this work. We propose to regularize the PSF by attracting it to a space of admissible PSFs, denoted as PSF-space. This is done by first performing example-based learning of the PSF-space by using linear principal components analysis (PCA) [49] or kernel PCA (KPCA) [57]. We then introduce into the deconvolution algorithm a term that attracts the PSF toward PSF-space.

\subsection{The Inspiration: Segmentation with Shape Priors}

The inspiration for the proposed approach stems from the prolific activity in the field of statistical shape priors for segmentation using active contours in the last decade [13], [14], [19], [20], [38], [54], [64] and, specifically, our work closely follows the methods presented in [12], [13], [14]. In those methods of segmentation, a contour is evolved over the image domain under the influence of an iterative process, usually aimed to optimize some cost functional. The latter is designed in such a manner that at convergence, the contour will delineate an object of interest within the image.

Due to noise, clutter, and occlusions, it is not always sufficient to use the given imagery data for the contour evolution. Therefore, a significant amount of research has been conducted in order to include into the contour evolution process prior information regarding the shape of the object of interest.

All of the above referenced methods operate in a similar outline: First, the space of shapes is learned from a training set of examples. Then, during the iterative process, a regularization term which attracts the contour under evolution toward the previously learned shape-space is included within the contour evolution process.

We make a direct analogy between those methods and the $\mathrm{BD}$ problem by replacing the shapes of interest in the active contour framework with the functions which are of our interest- - the PSFs. In the sequel, we will show how we adapt and apply the methods previously developed for constructing shape priors for segmentation methods as PSF priors for BD.

Finally, we would like to note that the BD problem is inherently free of a problem related to shape priors in the active contours framework, which is the problem of geometrical shape alignment and scaling. In the BD problem, we regularize functions and not shapes. Therefore, the PSFs are inherently aligned and scaled with respect to a global set of axes. 


\subsection{Integration as a Prior}

As we show later, we use learning methods in order to learn the PSF-space, prior to the deconvolution process. Then, at the end of each iteration $k$, we find the best approximation of the PSF estimate $\hat{h}_{k}$ within PSF-space. This approximation is denoted as $\mathcal{A}\left(\hat{h}_{k}\right)$.

In order to use $\mathcal{A}\left(\hat{h}_{k}\right)$ for PSF regularization, we propose a soft projection stage to be carried after each iteration:

$$
\mathcal{P}(h)=\eta \mathcal{A}(h)+(1-\eta) h,
$$

where $0 \leq \eta \leq 1$. The soft projection operation can be regarded as a weighted average of $h$ and $\mathcal{A}(h)$, which draws $h$ toward its approximation.

The complete iterative scheme we use, including the integrated denoising stage, is summarized as follows:

$$
\begin{aligned}
R_{k} & =g-\hat{f}_{k} * \hat{h}_{k}, \\
\bar{R}_{k} & =\operatorname{Denoise}\left(R_{k}\right), \\
\hat{f}_{k+1} & =\hat{f}_{k} \cdot\left[\left(\frac{\hat{f}_{k} * \hat{h}_{k}+\bar{R}_{k}}{\hat{f}_{k} * \hat{h}_{k}}\right) * \hat{h}_{k}^{s}\right], \\
\hat{h}_{k+1}^{\text {temp }} & =\frac{\hat{h}_{k}}{\sum_{\mathbf{x} \in \boldsymbol{\Omega}} \hat{f}_{k}(\mathbf{x})} \cdot\left[\left(\frac{\hat{f}_{k} * \hat{h}_{k}+\bar{R}_{k}}{\hat{f}_{k} * \hat{h}_{k}}\right) * \hat{f}_{k}^{s}\right], \\
\hat{h}_{k+1} & =\eta \mathcal{A}\left(\hat{h}_{k+1}^{\text {temp }}\right)+(1-\eta) \hat{h}_{k+1}^{\text {temp }} .
\end{aligned}
$$

\subsection{Statistical Learning Methods}

In the following section, we review two learning methods which we use in order to construct the PSF-space and the PSF approximation $\mathcal{A}(h)$. We use PCA [49] and KPCA [57] in order to learn the PSF-space. We note that PCA can be considered as a particular case of KPCA. However, for the sake of clarity, we present those methods separately.

\subsubsection{Principal Components Analysis}

PCA is a vector space transform which, for a given data set, finds a transformation of the axes in such a manner that the greatest variance by any projection of the data comes to lie on the first axis (called the first principal component), the second greatest variance on the second axis, and so on. Once this transform is calculated, the data are represented by the first $l$ principal directions, which are assumed to capture most of the variance in the data. It can be shown [49] that the principal directions are in fact the eigenvectors of the data covariance matrix. The number $l$ of principal directions to be used is usually determined by inspecting the eigenspectrum of the covariance matrix.

This approach is especially useful when the data is of very high dimension, but is known to have only a few degrees of freedom. This is exactly the case in the problem we have at hand. Our sampled PSFs have a very large number of voxels we need to estimate, and we can view them as very high-dimensional vectors. However, we know that the PSF has no more than a few degrees of freedom, as PSFs representing physical imaging systems are usually determined by a small number of parameters. By using PCA, we assume that the few degrees of freedom of the PSFs can be represented by a subspace of this high dimensional vector space. We note that this assumption is in general not true, as we cannot assure that an arbitrary
PSF can be represented as a linear combination of other PSFs. We further discuss this assumption and propose a method of relaxing it in the sequel.

In what follows, we assume that we have a training set of $N$ sampled PSFs $\left\{h_{1}, h_{2}, \ldots, h_{N}\right\}$, each containing $n_{v}$ voxels. Those training samples are assumed to represent PSFs of the imaging system used to acquire the images we wish to restore.

First, the mean PSF $\bar{h}$ is computed by taking the mean of the training PSFs $\bar{h}=\frac{1}{N} \sum_{k=1}^{N} h_{k}$. Next, we compute the covariance matrix $\mathbf{C}$ as follows: The mean PSF is subtracted from all training PSFs to create the centered training PSFs $\tilde{h}_{k}=h_{k}-\bar{h}$, and the latter are lexicographically ordered as column vectors. Each centered training sample is placed as the $k$ th column of an $n_{v} \times N$ matrix $\mathbf{A}=\left[\tilde{h}_{1}, \tilde{h}_{2}, \ldots, \tilde{h}_{N}\right]$. The $n_{v} \times n_{v}$ symmetric covariance matrix is defined as $\mathbf{C}=\frac{1}{N} \mathbf{A} \mathbf{A}^{\mathrm{T}}$. Then, an eigendecomposition is performed on $\mathbf{C}, \mathbf{C}=\mathbf{U} \boldsymbol{\Sigma} \mathbf{U}^{\mathrm{T}}$, where $\mathbf{U}$ is a matrix whose columns are the eigenvectors of $\mathbf{C}$ (the principal components) and $\boldsymbol{\Sigma}$ is a diagonal matrix with the corresponding eigenvalues of $\mathbf{C}$ on its diagonal, in descending order.

We denote by $u_{k}$ the $k$ th principal component. The principal components are sampled eigen-PSFs, which can be used as a basis for representation of other PSFs. We denote by $\mathbf{U}_{l}$ the $n_{v} \times l$ matrix composed of the first $l$ columns of $\mathbf{U}$. The coordinates of the projection of an arbitrary test function ${ }^{3} h$ onto the space of PSFs spanned by the first $l$ principal components can be computed as

$$
\alpha^{l}=\mathbf{U}_{l}^{T}(h-\bar{h}) .
$$

And the projection itself can be calculated as

$$
\mathcal{P}^{l}(h)=\sum_{k=1}^{l} \alpha_{k}^{l} u_{k}+\bar{h},
$$

where $\alpha_{k}^{l}$ is the $k$ th component of the vector $\alpha^{l}$. It can be shown [49] that the error of representing any of the training set examples $h_{k}$ by their projection $\mathcal{P}^{l}\left(h_{k}\right)$ is minimal in the least square sense.

It is important to point out that, during the derivation of PCA, it is assumed that any PSF within the data set, and essentially within the PSF-space, can be represented as a linear combination of some basis (a vector space assumption). As previously noted, this assumption is in general not true, as a PSF cannot be represented in general as a linear combination of the sample PSFs. In spite of this, this assumption can be made as a rough approximation, especially when an arbitrary PSF is close to the training set in the sense of small variations around its mean.

Finally, we take the approximation $\mathcal{A}(\cdot)$ in (7) simply to be the projection $\mathcal{P}^{l}(\cdot)$, i.e.,

$$
\mathcal{A}\left(\hat{h}_{k+1}^{t e m p}\right)=\mathcal{P}^{l}\left(\hat{h}_{k+1}^{t e m p}\right) .
$$

\subsubsection{Kernel PCA}

In order to relax the vector space assumption which is made when using the PCA method, we propose using Kernel

3. Which is assumed to be sampled in the same scheme in which the training PSFs were sampled. 
PCA. Kernel PCA can be considered as a nonlinear extension of PCA. KPCA was first introduced in [44], [57], where it was proven to be a powerful technique for extracting the nonlinear structure of a data set.

In KPCA, the data is first mapped from the original input space $\mathcal{I}$, where it resides, to some feature space $\Gamma$, via a nonlinear function $\varphi$. Then, linear PCA is employed in feature space to find the feature space principal components which correspond to the largest variations of the data set in feature space. Similarly to the linear PCA case, we use the first $l$ principal components, which account for the largest variation possible within the data set using $l$ directions.

Due to the nonlinearity of the mapping $\varphi$, this technique enables us to capture nonlinear variations within the data set. In essence, it relaxes the assumption that an arbitrary PSF can be represented as a linear combination of the training set PSFs. More specifically, by using KPCA, we assume that the above assumption holds in feature space, thus allowing the representation of an arbitrary PSF as some nonlinear function of the training set PSFs.

In general, the feature space can be of very high dimension [44], and in practice, we do not necessarily know which transformation $\varphi$ will provide useful results. In order to overcome those limitations, the mapping $\varphi$ is not carried out in explicit form. Moreover, $\varphi$ is usually not known and all calculations are carried out by the use of Mercer kernels. A Mercer kernel is a function $k: \mathcal{I} \times \mathcal{I} \rightarrow \mathbb{R}$, which for all points in the data set $k\left(h_{i}, h_{j}\right)$ is positive and symmetric with respect to its arguments, i.e., $k\left(h_{i}, h_{j}\right)=k\left(h_{j}, h_{i}\right)$. It has been shown [4] that applying the kernel in input space is equivalent to the calculation of an inner product in feature space:

$$
k\left(h_{i}, h_{j}\right)=\left\langle\varphi\left(h_{i}\right), \varphi\left(h_{j}\right)\right\rangle .
$$

Therefore, all calculations that can be formulated in terms of inner products in feature space, can be carried out in input space without explicitly using the mapping $\varphi$, and only the kernel $k(\cdot, \cdot)$ has to be known.

The choice of the kernel can be arbitrary as long as it satisfies the conditions of symmetry and positivity. One especially popular choice for a kernel which was also proven to be useful [44], [13], [14] is the exponential kernel

$$
k\left(h_{i}, h_{j}\right)=\exp \left(-\frac{\left\|h_{i}-h_{j}\right\|^{2}}{2 \sigma^{2}}\right),
$$

where $\sigma$ is a parameter of the kernel function. Due to its successful application for different problems, we choose to use the exponential kernel in what follows. Intuitively, it can be understood that this type of kernel provides some kind of adjacency measure between two PSFs, i.e., it measures the similarity between PSFs. Therefore, it is not surprising that the analysis of such a kernel over the training set is capable of capturing information regarding the relations between the PSFs in the training set. This property of the kernel function also explains the advantage of choosing the kernel function over choosing the mapping $\varphi$, which is more difficult to obtain intuition about.

In the following, we briefly describe the KPCA method [35], [44] while closely following the notations in [44]. As in the linear PCA formulation, we assume having a training set of $N$ sampled PSFs $\left\{h_{1}, h_{2}, \ldots, h_{N}\right\}$, each one containing $n_{v}$ voxels. The symmetric positive definite kernel matrix is defined as $\mathbf{K}_{i j}=k\left(h_{i}, h_{j}\right)$. We define the mean map of the training data set in feature space $\bar{\varphi}=\frac{1}{N} \sum_{k=1}^{N} \varphi\left(h_{k}\right)$ and the centered maps of the data set in feature space as $\tilde{\varphi}\left(h_{k}\right)=\varphi\left(h_{k}\right)-\bar{\varphi}$. Then, the centered kernel function is defined as $\tilde{k}\left(h_{i}, h_{j}\right)=\left\langle\tilde{\varphi}\left(h_{i}\right), \tilde{\varphi}\left(h_{j}\right)\right\rangle$ and the centered kernel matrix as $\tilde{\mathbf{K}}_{i j}=\tilde{k}\left(h_{i}, h_{j}\right)$. It can be easily shown that the matrix $\mathbf{K}$ can be centered as follows: $\tilde{\mathbf{K}}=\mathbf{H K H}$, where $\mathbf{H}=\mathbf{I}_{N}-\frac{1}{N} \mathbf{1}_{\mathrm{N}} \mathbf{1}_{\mathrm{N}}^{\mathrm{T}} . \mathbf{I}_{N}$ Denotes the $N \times N$ unit matrix and $\mathbf{1}_{\mathrm{N}}$ is an $N \times 1$ column vector of ones.

Eigenvalue decomposition is performed for $\tilde{\mathbf{K}}$ : $\tilde{\mathbf{K}}=$ $\mathbf{V} \boldsymbol{\Lambda} \mathbf{V}^{\mathrm{T}}$ where $\mathbf{V}$ is a matrix whose columns are the eigenvectors of $\tilde{\mathbf{K}}$ (the principal components) and $\boldsymbol{\Lambda}$ is a diagonal matrix with the corresponding eigenvalues of $\tilde{\mathbf{K}}$, denoted as $\left\{\lambda_{1}, \lambda_{2}, \ldots, \lambda_{N}\right\}$ on its diagonal, in descending order. We denote by $v_{k}$ the $k$ th principal component of the kernel matrix.

We note that it follows from (11) that the kernel matrix $\tilde{\mathbf{K}}$ is closely related to the covariance matrix in feature space and it can be shown [57] that the $k$ th eigenvector of the feature space covariance matrix can be expressed as:

$$
w_{k}=\frac{1}{\sqrt{\lambda_{k}}} \sum_{i=1}^{N} v_{k, i} \tilde{\varphi}\left(h_{i}\right)=\frac{1}{\sqrt{\lambda_{k}}} \tilde{\varphi}^{\mathrm{T}} v_{k}
$$

where $v_{k, i}$ is the $i$ th component of the vector $v_{k}$ and $\tilde{\varphi}=\left[\tilde{\varphi}\left(h_{1}\right), \tilde{\varphi}\left(h_{2}\right), \ldots, \tilde{\varphi}\left(h_{N}\right)\right]^{T}$.

The coordinates of the projection of the mapping of an arbitrary test function $\varphi(h)$ onto the space spanned by the first $l$ principal components can be computed as

$$
\gamma_{k}=\left\langle\tilde{\varphi}(h), w_{k}\right\rangle=\frac{1}{\sqrt{\lambda_{k}}} \sum_{i=1}^{N} v_{k, i} \tilde{k}\left(h, h_{i}\right),
$$

where $\tilde{k}\left(h, h_{i}\right)=\left\langle\tilde{\varphi}(h), \tilde{\varphi}\left(h_{i}\right)\right\rangle$ can be calculated as [44]:

$$
\tilde{k}\left(h, h_{i}\right)=k\left(h, h_{i}\right)-\mathbf{1}_{\mathrm{N}}^{\mathrm{T}} k_{h}-\mathbf{1}_{\mathrm{N}}^{\mathrm{T}} k_{h_{i}}+\frac{1}{N^{2}} \mathbf{1}_{\mathrm{N}}^{\mathrm{T}} \mathbf{K} \mathbf{1}_{\mathrm{N}}
$$

with $k_{x}=\left[k\left(x, h_{1}\right), k\left(x, h_{2}\right), \ldots, k\left(x, h_{N}\right)\right]^{T}$. We note that $k_{h_{i}}=\left[k\left(h_{i}, h_{1}\right), k\left(h_{i}, h_{2}\right), \ldots, k\left(h_{i}, h_{N}\right)\right]^{T}$ is simply the $i$ th column of $\mathbf{K}$.

Finally, the projection of $\varphi(h)$ onto the subspace of feature space spanned by the first $l$ eigenvectors is given by:

$$
\begin{aligned}
\mathcal{P}^{l}(\varphi(h)) & =\sum_{k=1}^{l} \gamma_{k} w_{k}+\bar{\varphi} \\
& =\sum_{k=1}^{l} \frac{1}{\lambda_{k}}\left(v_{k}^{T} \tilde{k}_{h}\right)\left(\tilde{\varphi}^{T} v_{k}\right)+\bar{\varphi} \\
& =\tilde{\varphi}^{\mathrm{T}} \mathbf{M} \tilde{k}_{h}+\bar{\varphi}
\end{aligned}
$$

where $\mathbf{M}=\sum_{k=1}^{l} \frac{1}{\lambda_{k}} v_{k} v_{k}^{T}$.

It is noteworthy that PCA is merely a particular case of KPCA with $k\left(h_{i}, h_{j}\right)=\left\langle h_{i}, h_{j}\right\rangle$. In essence, applying the nonlinear kernel prior to the extraction of principal components enables us to capture nonlinear structure of the training data set and avoids the vector space assumption pointed out earlier. 


\subsubsection{The Pre-Image Problem}

We note that the expression for the KPCA projection (15) is given in feature space. Since the formulation in (7) requires an approximation $\mathcal{A}(h)$ which lies in input space, we are interested in obtaining the pre-image of $\mathcal{P}^{l}(\varphi(h))$, i.e., a function $h^{P I}$ in input space that satisfies

$$
\varphi\left(h^{P I}\right)=\mathcal{P}^{l}(\varphi(h))
$$

In general, the pre-image does not necessarily exist [44], [51]. However, methods for its estimation have been previously proposed [44], [51]. We choose to use the method described in [51] due to its elegance, ease of computation, and closed form formulation. This formulation, which is given in detail in [51], produces the following scheme for the calculation of the preimage of $\mathcal{P}^{l}(\varphi(h))$ :

1.

$$
\mu_{i}=\sum_{k=1}^{l} \gamma_{k} v_{k, i}, \quad \tilde{\mu}_{i}=\mu_{i}+\frac{1}{N}\left(1-\sum_{j=1}^{N} \mu_{j}\right) .
$$

2.

$$
\begin{aligned}
& \left\|\mathcal{P}^{l}(\varphi(h))-\varphi\left(h_{i}\right)\right\|^{2} \\
& =\left(k_{h}+\frac{1}{N} \mathbf{K} \mathbf{1}_{\mathrm{N}}-2 k_{h_{i}}\right)^{T} \mathbf{H M H}\left(k_{h}-\frac{1}{N} \mathbf{K} \mathbf{1}_{\mathrm{N}}\right) \\
& \quad+\frac{1}{N^{2}} \mathbf{1}_{\mathrm{N}}^{\mathrm{T}} \mathbf{K} \mathbf{1}_{\mathrm{N}}+\mathbf{K}_{i i}-\frac{2}{N} \mathbf{1}_{\mathrm{N}}^{T} k_{h_{i}} .
\end{aligned}
$$

3.

$$
h^{P I} \approx \frac{\sum_{i=1}^{N} \tilde{\mu}_{i} h_{i}\left(1-\frac{1}{2}\left\|\mathcal{P}^{l}(\varphi(h))-\varphi\left(h_{i}\right)\right\|^{2}\right)}{\sum_{i=1}^{N} \tilde{\mu}_{i}\left(1-\frac{1}{2}\left\|\mathcal{P}^{l}(\varphi(h))-\varphi\left(h_{i}\right)\right\|^{2}\right)} .
$$

We summarize the overall process of obtaining a preimage of the feature space projection of an arbitrary input space test function using the KPCA method for an exponential kernel:

\section{Learning stage}

1. Calculate the kernel matrix $\mathbf{K}_{\mathbf{i j}}=k\left(h_{i}, h_{j}\right)=$ $\exp \left(-\frac{\left\|h_{i}-h_{j}\right\|^{2}}{2 \sigma^{2}}\right)$.

2. Center the kernel matrix $\tilde{\mathbf{K}}=\mathbf{H K H}$.

3. Preform eigenvalue decomposition $\tilde{\mathbf{K}}=\mathbf{V} \boldsymbol{\Lambda} \mathbf{V}^{\mathrm{T}}$.

Projection pre-image

1. Calculate the projection coefficients $\gamma_{k}$ by (13).

2. Calculate the coefficients $\mu_{i}$ according to (17).

3. Calculate $\left\|\mathcal{P}^{l}(\varphi(h))-\varphi\left(h_{i}\right)\right\|^{2}$ by $(18)$.

4. Calculate the estimated preimage $h^{P I}$ by (19).

We take the approximation $\mathcal{A}(\cdot)$ in (7) simply to be the pre-image estimate $h^{P I}$, i.e.,

$$
\mathcal{A}\left(\hat{h}_{k+1}^{t e m p}\right)=\hat{h}_{k+1}^{P I} .
$$

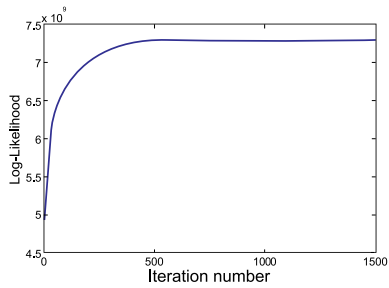

(a)

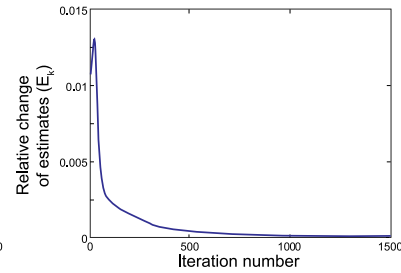

(b)
Fig. 1. Convergence of the iterative process. (a) Log-likelihood function. (b) Relative change of estimates.

Finally, we would like to note that the approximation $\mathcal{A}(\cdot)$ for both the PCA and KPCA methods requires no more than a few matrix and vector products and thus the projection stage is very efficient.

\section{Convergence Properties}

It is a well-known result that each step of the alternating minimization scheme (4) by itself converges to a global optimum [10]. Therefore, it is guaranteed that this iterative process yields a sequence of estimates with nondecreasing log-likelihood. In addition, other researchers provide convergence proofs for specific classes of priors, see, e.g., [17].

However, since we use the formulation in (7) which includes both the residual denoising step and our prior term, there is no guarantee that the iterative scheme we propose brings (3) even to a local optimum.

In practice, we observed that the iterative scheme (7) produces sequences of estimates with nondecreasing likelihood, except for the first few iterations, in some data sets. Since the calculation of the log-likelihood requires additional computations at each iteration, we define the relative change of estimates at iteration $k$ as

$$
E_{k}=\frac{1}{2}\left(\frac{\left\|\hat{h}_{k}-\hat{h}_{k-1}\right\|}{\left\|\hat{h}_{k-1}\right\|}+\frac{\left\|\hat{f}_{k}-\hat{f}_{k-1}\right\|}{\left\|\hat{f}_{k-1}\right\|}\right)
$$

and stop the iterative scheme when $E_{k}$ drops below a predetermined threshold. Typical plots of the log-likelihood and the relative change of estimates along the iterative process are displayed in Fig. 1.

\section{Application for Wide-Field Fluorescence MICROSCOPY}

\subsection{Background}

Wide-field fluorescence microscopy (WFFM) is a modality used for imaging 3D biological specimens. The microscope objective lens is immersed in some oily medium, ${ }^{4}$ denoted as the immersion medium. A cover slide of the same refractive index as the immersion medium is mounted below the layer of oil and covers the imaged specimen. The microscope optics are focused at a plane of certain depth within the imaged sample, denoted as the in focus plane, and produce a $2 \mathrm{D}$ image, of a section within the sample. By consecutively focusing the microscope at different depths within the sample, a collection of $2 \mathrm{D}$ sections is formed, overall

\footnotetext{
4. This layer of oil enhances the optical properties of the microscope.
} 
creating a 3D image. The horizontal and vertical directions spanning the 2D in-focus planes are denoted as $x$ and $y$, respectively. The coordinate which varies along the optical axis of the microscope (the depth coordinate) is denoted as $z$. This process is named optical sectioning [42]. The detector array receives a significant amount of radiation originating from out of focus planes, in addition to the radiation coming from the in focus plane. The latter phenomenon results in significant blurring of the acquired images and thus deteriorates image quality. In addition, WFFM imagery is usually contaminated by photon noise.

In many imaging systems, the PSF is determined solely by the properties of the imaging system. Therefore, under this assumption, knowledge of the imaging system implies exact knowledge of the PSF or at least a good approximation of it. In microscopy, however, the imaged sample itself influences the PSF and therefore exact a priori knowledge of the PSF is not available, hence the necessity for blind deconvolution algorithms. Moreover, as we will see next, the WFFM PSF possesses some properties which make the restoration process especially difficult.

\subsection{PSF Model}

Several analytical models for the WFFM PSF have been proposed and validated [3], [24], [25], [27], [62], [63]. A comprehensive discussion of the WFFM optics is out of the scope of this work, and the interested reader is referred to the above references and the references therein. However, a PSF model was required for the proposed BD algorithm for the cause of simulating the training set of PSFs. The PSF model used for this work follows [62],[2]:

$$
\begin{aligned}
h(r, z)= & I_{0} \cdot \mid \int_{-\infty}^{\infty} \int_{-\infty}^{\infty} \operatorname{pupil}\left(k_{r}\right) \cdot \operatorname{defocus}\left(k_{r}, z\right) \\
& \left.\cdot \operatorname{depth}\left(k_{r}\right) \cdot e^{-2 \pi i\left(k_{x} x+k_{y} y\right)} d k_{x} d k_{y}\right|^{2}, \\
& \operatorname{pupil}\left(k_{r}\right)= \begin{cases}1, & k_{r} \leq \frac{N A}{\lambda_{0}} \\
0, & \text { else, }\end{cases} \\
& \operatorname{defocus}\left(k_{r}, z\right)=\exp \left\{i \cdot k_{0} \cdot z \cdot \cos \left(\theta_{0}\left(k_{r}\right)\right)\right\}, \\
\operatorname{depth}\left(k_{r}\right)= & \exp \left\{i \cdot k_{0} \cdot d \cdot\left[n_{p} \cos \left(\theta_{p}\left(k_{r}\right)\right)-n_{0} \cos \left(\theta_{0}\left(k_{r}\right)\right)\right]\right\} .
\end{aligned}
$$

Where $x, y, z$ are spatial Cartesian coordinates,

$$
\begin{aligned}
k_{r} & =\sqrt{k_{x}^{2}+k_{y}^{2}}, \quad r=\sqrt{x^{2}+y^{2}}, \theta_{0}\left(k_{r}\right)=\arcsin \left(\frac{\lambda_{0}}{n_{0}} k_{r}\right), \\
\theta_{p}\left(k_{r}\right) & =\arcsin \left(\frac{\lambda_{0}}{n_{p}} k_{r}\right), k_{0}=\frac{2 \pi n_{0}}{\lambda_{0}}
\end{aligned}
$$

is the wave frequency in the medium, $\lambda_{0}$ is the light wavelength, $N A$ is the numerical aperture of the microscope, $n_{0}$ is the refractive index of the immersion medium, $n_{p}$ is the refractive index of the imaged sample, $d$ is the depth of the in-focus plane below the cover glass, and $I_{o}$ is a scaling factor that was chosen so that $h$ will integrate to unity.

One important property of the WFFM PSF is that it is radially symmetric in the $\mathrm{x}-\mathrm{y}$ plane: ${ }^{5}$

5. This is a good assumption since in-plane aberrations are practically fully corrected for within the field imaged by the microscope in all high quality objective lenses.

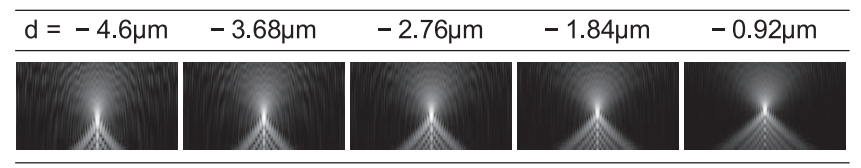

(a)

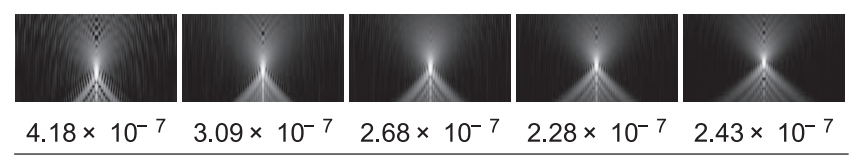

(b)

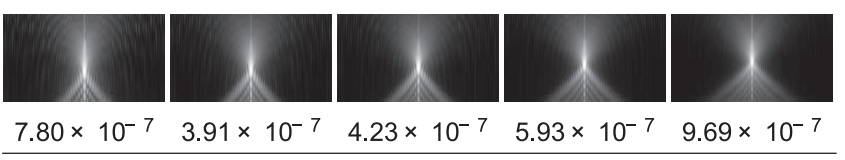

(c)

Fig. 2. Some of the PSFs used in the training set, along with their PSFspace approximations. $x-z$ sections are displayed in a logarithmic color map. (a) A few training set PSFs. The value of $d$ is displayed above each column. (b) Corresponding PCA space approximations. (c) Corresponding KPCA space approximations. The RMSE values (approximation error) are displayed below each approximation image.

$$
h(x, y, z)=h_{\text {rad }}\left(\sqrt{x^{2}+y^{2}}, z\right) \equiv h_{\text {rad }}(r, z) .
$$

The formulation in (21) also reveals the fact that in WFFM, the degradation model is shift variant, as it is dependent upon the depth of the in-focus plane within the immersion medium. However, the convolutive model is a very good approximation for the degradation induced by the microscope $^{6}$ and is often assumed in literature. By adopting this assumption, $d$ becomes the average depth of the imaged sample below the cover slide. Examples for various simulated PSFs can be observed in Fig. 2.

\subsection{Depth Aberrations}

The WFFM PSF is dependent on a few parameters, most of which relate to the imaging system itself. However, the differences between the refractive indices of the sample and the immersion oil affect the PSF as well. This effect manifests in the form of asymmetry of the PSF along the z-direction, as can be observed in Fig. 2. We term the spherical aberration caused by imaging into a specific depth $d$ below the cover slide for live biological specimens with a refractive index different than the immersion oil depth aberration. Since the depth aberrations are governed by parameters related to the imaged sample itself, they are the root cause for the inability of obtaining exact a priori knowledge of the PSF. In practice, all of the PSF parameters, with the exception of the sample depth $d$ and the refractive index of the sample $n_{p}$ are usually known. In the sequel, we will show how the known parameters can be utilized, and the unknown depth aberration can be estimated using our proposed method.

The WFFM PSF does not have a compact spatial domain support (see Fig. 2), which essentially means that many PSF voxels are required to be estimated, thus increasing the number of degrees of freedom within the system. In

6. This is true since the thickness of the imaged sample is negligible in comparison to the thickness of the immersion medium. 
addition, the optimization over the PSF cannot be easily carried out in parametric form, due to its complexity.

An approach that has the potential of overcoming those difficulties is to approximate the PSF with simple functions. Such an approach is proposed in [48] for blind deconvolution of confocal fluorescence microscopy images, where the PSF is approximated by a Gaussian function. However, it has been previously shown that in 3D WFFM, the PSF cannot be well approximated by Gaussians [69], even in the case of no depth aberrations.

From all of the above we conclude that, first, in WFFM it is essential to use blind deconvolution methods since the PSF is affected by the imaged sample itself and, second, that using example-based learning for studying the class of PSFs seems to be advantageous in WFFM since the PSF cannot be easily and accurately parametrized.

\section{Numerical EXPERIMENTS}

In order to validate the proposed BD scheme, we tested it on five data sets. The first two data sets were created by simulating a $128 \times 128 \times 128$ synthetic image depicting three tori of different sizes and intensities (Fig. 6a). The synthetic image was blurred by two different PSFs, one free of depth aberrations and one containing depth aberrations $(d=1 \mu \mathrm{m})$. Then, each blurred image was scaled to a maximum intensity value of 1,000, and corrupted by Poisson noise. ${ }^{7}$ In addition, three images of biological specimens were acquired by a WFFM. Two of the acquired images were known to have depth aberrations and one was known to be free of depth aberrations or to have very little depth aberration. ${ }^{8}$

The original images were of size $1,024 \times 1,024$ in the $x-y$ plane and contained 42 to 46 sections in the $z$-direction. The pixel size is $0.0663 \mu \mathrm{m}$ in the $x-y$ plane and $0.2 \mu \mathrm{m}$ along the $z$-axis. In order to facilitate the processing of such large data sets, a $256 \times 256$ section was cropped from each image. The images were not cropped in the $z$-direction.

Each of the images was processed using three different methods: BD using (7) with the PCA and KPCA priors, and the AIDA algorithm depicted in [30]. In addition, the synthetic images were processed by nonblind deconvolution using (5), excluding the last step, with the true PSF kept fixed.

\subsection{Implementation Details}

In the following experiments, it was assumed that the microscope parameters are known and the only unknown parameter is the sample depth $d$. As previously discussed in Section 6.3 , in practice neither $n_{p}$ nor $d$ are exactly known, but since they both control the depth aberrations in a similar manner, for the following experiments it is sufficient to modify only one of them. This is a practical assumption and we stress that it does not reduce the generality of the proposed method.

In general, it is possible to create the training set of PSFs by imaging small fluorescent beads, see, e.g., [30], [56].

7. The scaling of the blurred image determines the noise magnitude.

8. The degree of depth aberrations was controlled in this experiment by replacing the objective immersion oil with media of different refractive indices.
However, since it is beneficial for our method to use a large number of PSFs for the training set, and the acquisition of a large number of samples under varying acquisition conditions is a tedious and cumbersome task, we used simulated PSFs for our experiments. The training set contained a total of 201 PSFs which were simulated according to the formulation (21) with $d$ varying from $-4.6 \mu \mathrm{m}$ to $4.6 \mu \mathrm{m}$, which covers the range of expected depth aberrations. A sample of the training set PSFs is displayed in Fig. 2, along with their best approximations by PCA and KPCA. As a measure of approximation error we calculated the root mean squared error (RMSE). We define the RMSE as

$$
\operatorname{RMSE}(f, \hat{f})=\left(\frac{1}{|\Omega|} \sum_{\mathbf{x} \in \Omega}(f(\mathbf{x})-c \cdot \hat{f}(\mathbf{x}))^{2}\right)^{\frac{1}{2}},
$$

where $|\Omega|$ is the number of voxels in the image and

$$
c=\frac{\sum_{\mathbf{x} \in \Omega} f(\mathbf{x})}{\sum_{\mathbf{x} \in \Omega} \hat{f}(\mathbf{x})}
$$

accounts for different scaling between the images. ${ }^{9}$ It can be observed both visually and by examining the RMSE values, that the PSF-space approximation error is low for both the PCA and KPCA methods. It can also be seen in Fig. 2 that the KPCA method provides smoother approximations than PCA, with higher RMSE values. This is due to the choice of the KPCA parameter $\sigma$, which is set to a value sufficiently large for providing good approximations for PSFs which do not belong to the training set. The influence of $\sigma$ is further discussed in the following.

In addition, since the implementation of the learning stage depicted in Section 4.3 requires the storage of the entire training set, in general it requires a very large amount of memory space. However, in WFFM, we can take advantage of our prior knowledge of the PSF radial symmetry, and perform the entire statistical learning stage on a $2 \mathrm{D}$ half section of the $r-z$ plane, thus significantly reducing memory requirements by an order of magnitude. Exploiting the PSF radial symmetry also facilitates the diagonalization of the covariance matrix in the PCA case, which would be otherwise infeasible.

Another implementation issue is the choice of parameters, namely $\eta$ and $\sigma$. The regularization parameter $\eta$ controls the degree in which the PSF estimates are drawn to the PSF-space. It can be understood from (6) that $\eta=1$ implies a hard constraint, where the PSF is projected onto PSF-space at the end of each iteration. Since the MLEM iterative process is very sensitive to local maxima, we found this approach not to be useful. During our experimentation, it was empirically found that many PSFs in the training set correspond to local optima of the iterative process. At the beginning of the iterative process, the PSF estimates might be far from the optimal solution. The hard constraint forces all PSF estimates to reside within the learned PSF-space, and might cause the iterative process to be attracted to a local maximum. We demonstrate this property of the deconvolution process in Fig. 3. Nonblind deconvolution was carried

9. It can be easily shown that this choice of the scaling factor $c$ minimizes the mean square error. 


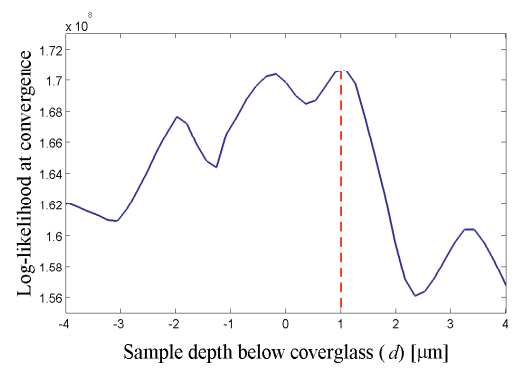

Fig. 3. Influence of sample depth on log-likelihood values. The vertical dashed line marks the true value of $d$, at $1 \mu \mathrm{m}$.

out on the synthetic image shown in Fig. 6g, using 45 different PSFs, with $d$ varying from $-4 \mu \mathrm{m}$ to $4 \mu \mathrm{m}$. The log-likelihood values at convergence were recorded and displayed against the value of $d$. It can be observed that although the global maximum is obtained very close to the true solution $d=1 \mu \mathrm{m}$, a number of other local maxima exist as well. Setting $\eta<1$ allows the PSF estimates to approach this global maximum on a different path, which could potentially avoid the local maxima. On the other hand, setting $\eta$ to a very small value might lead to insufficient regularization. We found the adequate values of $\eta$ to reside between 0.01 and 0.1 , and that the results do not vary significantly when $\eta$ is set to a value within this range.

The KPCA parameter $\sigma$ controls the kernel width and ultimately determines the degree of mixing allowed between the training set PSFs when creating the PSF-space approximation $\mathcal{A}(\hat{h})$ [13], [14]. As the value of $\sigma$ grows, more training samples are weighted into the PSF approximation, causing the PSF approximation to become blurry and less plausible. Contrary to that, setting $\sigma$ to extremely low values causes the regularization scheme to degenerate to a nearest-neighbor search within the training set. This is usually not desirable, as the true PSF is not expected to reside within the training set. We suggest using the values of the distances between the training set samples $\left\|h_{i}-h_{j}\right\|$ as a guide to the value of $\sigma$.

All convolution operations were carried out using the fast Fourier transform (FFT). Running on the $128 \times 128 \times$ 128 data set using the KPCA regularization, a single iteration (requiring three convolution operations) took approximately $14 \mathrm{~s}$ on an Intel Q6600 $2.4 \mathrm{GHz}$ processor (utilizing a single core) and exhibited peak memory usage of $750 \mathrm{Mb}$. In our experiments, the algorithm typically converged in 500-600 iterations, yielding a total processing time of around $2 \mathrm{~h}$. Out of the total processing time, the PSF regularization code took about 20 percent. The FFT code took most of the remaining processing time $(\sim 70$ percent of total processing time).

\subsection{Results}

The images were processed according to (7), with the PSF regularization implemented according to (6) with $\eta=0.05$, for both the PCA and KPCA methods. In the KPCA case, $\sigma$ was set to the 0.2 quantile of the distances $\left\|h_{i}-h_{j}\right\|$ within the training set. The value of $l$, which is the number of principal components used, was set to 10 for both the PCA and the KPCA methods. These values were determined by observing the eigen-spectrum of the covariance matrix in the PCA case, and the centered kernel matrix in the KPCA



(a)

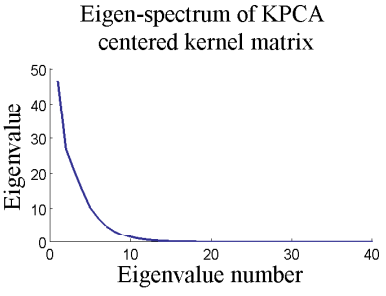

(b)
Fig. 4. Selection of the number of used eigenvalues for PCA and KPCA methods. (a) Eigen-spectrum of covariance matrix. (b) Eigen-spectrum of centered kernel matrix.

case, as can be observed in Fig. 4. The PCA eigenvalues corresponding to the 10 largest magnitude eigenvalues are displayed in Fig. 5.

We note that the observed reconstruction results were found not to be highly dependent on the value of $l$ and, in practice, any number between 5 and 30 performed well, for both methods. The algorithm was initialized with the acquired image as the object estimate and a symmetrical PSF support as the PSF estimate (see Figs. 7, 8, 9).

As previously mentioned, the AIDA algorithm, which we compare our results to, uses a PSF prior given by the user. In the following experiments, we supplied AIDA with a symmetrical PSF as the prior.

As a measure of fidelity to the ground truth image, we calculated the RMSE between the object estimates and the original image according to (23). We note that the maximal intensity value in the ground truth image is 1,000. In addition, the RMSE between the estimated PSFs and the true PSFs was calculated.

Results for the synthetic object are shown in Fig. 6. For the nonaberrated PSF (Fig. 6b, 6c, 6d, 6e, 6f), both PCA and KPCA regularizations achieve results which are very similar to the nonblind deconvolution results. This can be observed both visually and by examining the RMSE values which appear below the images. For the more challenging case of a depth aberrated PSF (Figs. 6g, 6h, 6i, 6j, 6k), both regularization methods fall short of the nonblind deconvolution results. It is also noteworthy that KPCA regularization performs somewhat better than PCA. AIDA results for the synthetic image seem very smooth and visually appealing. This effect stems from the object gradient minimizing regularization term it uses. However, despite the fact that the ground truth image complies very well with the gradient minimization prior, AIDA performs worse than the proposed method in quantitative terms, as can be deduced from the RMSE values. In addition, observing the PSF estimate produced by AIDA for the

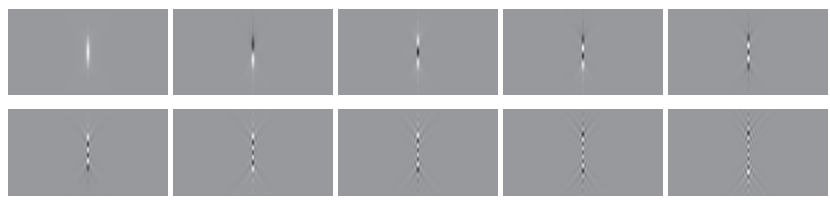

Fig. 5. The PCA eigenvectors corresponding to the 10 largest magnitude eigenvalues. The eigenvectors are displayed in descending order of the corresponding eigenvalues, from top left to bottom right. 


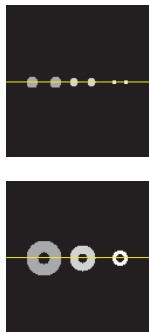

RMSE
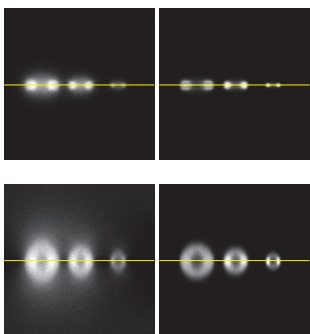

35.81

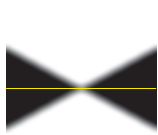

3.56

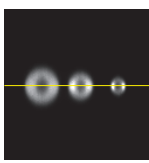

19.92

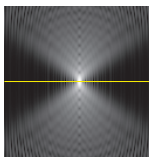

0
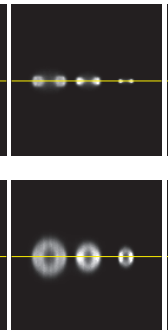

19.26



1.46
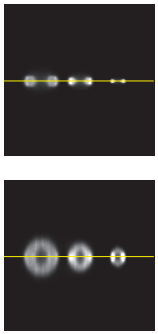

19.30

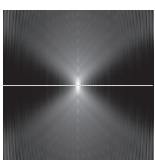

1.1
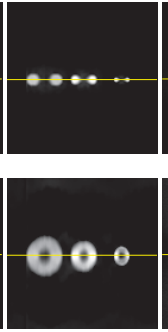

25.47



5.12
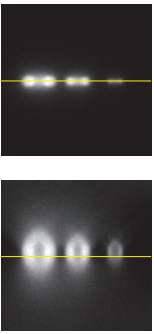

36.16

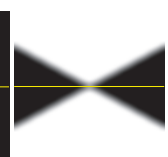

3.25
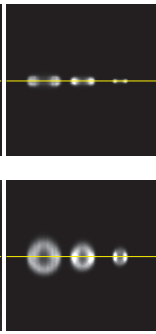

20.11

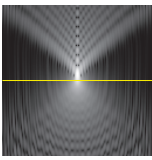

0

(h)

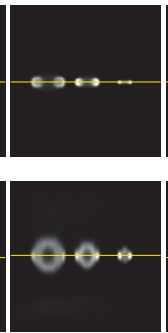

26.92

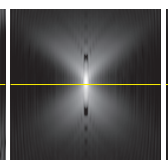

4.68

(i)

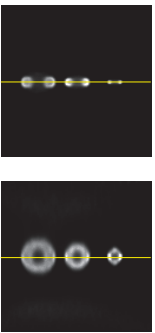

24.88

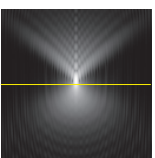

3.22

(j)
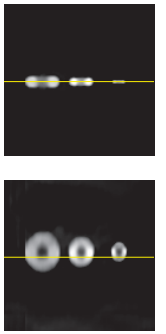

31.23

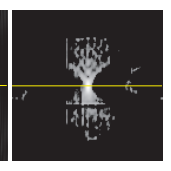

7.23

(k)

Fig. 6. Blind deconvolution results for the synthetic image. (a) Original image. (b) Corrupt image without depth aberrations. (c) Results using nonblind deconvolution. (d) Results using PCA regularization. (e) Results using KPCA regularization. (f) Results using AIDA. (g) Corrupt image with depth aberrations. (h) Results using nonblind deconvolution. (i) Results using PCA regularization. (j) Results using KPCA regularization. (k) Results using AIDA. Top row: $x-y$ image section. Second row: $x-z$ image section. Locators indicate the location of displayed sections. Third row: RMSE between each image and the original image in (a). Fourth row: $r-z$ PSF section, logarithmic scaling. Locators indicate $z=0$. PSF initial estimate is displayed in (b) and (g). Fifth row: RMSE between each PSF and the corresponding true PSF in (c) and (h).

depth aberrated case (Fig. 6k), it clearly fails to detect the depth aberrations.

Results for the real world depth aberrated images are shown in Fig. 7 and Fig. 8. Results for the real world aberration free image are shown in Fig. 9.

It can be seen that the proposed algorithm significantly enhances image resolution, as many new details can be resolved in the reconstructed images. In addition, the reconstructed images seem free of noise. We further observe that the proposed algorithm succeeds in estimating the depth aberrations of the PSF, as the PSFs estimated by the algorithm are indeed asymmetric w.r.t the $z$-axis in the images acquired
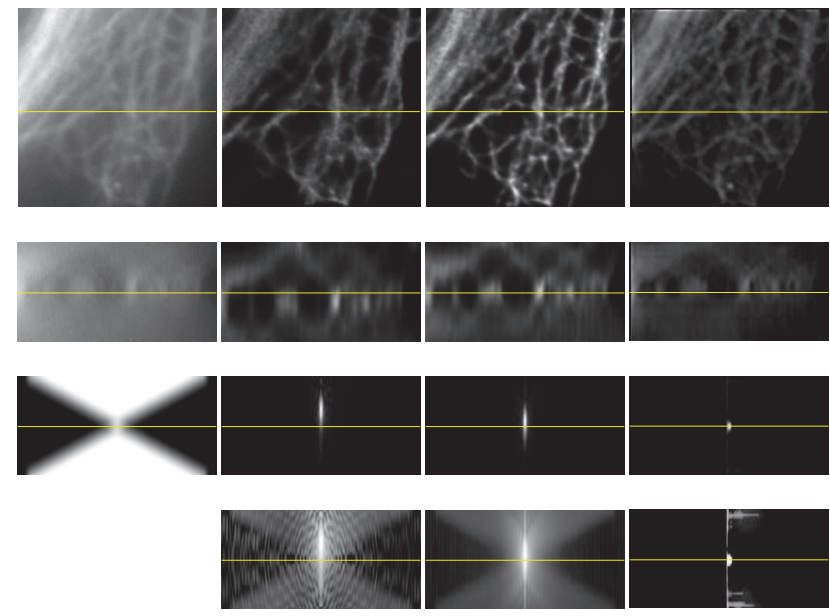

(a)

(b)

(c)

(d)

Fig. 7. Blind deconvolution results for a real depth aberrated image. (a) Original image. (b) Results using PCA regularization. (c) Results using KPCA regularization. (d) Results using AIDA. Top row: $x-y$ image section, second row: $x-z$ image section. PSF initial estimate is displayed in (a). Locators indicate the location of displayed sections. Third row: $r-z$ PSF section, linear scaling. Fourth row: $r-z$ PSF section, logarithmic scaling. Locators indicate $z=0$. in the setting which contained the aberrations (Figs. 7 and 8), and symmetric for the aberration free setting (Fig. 9). We note that once again, AIDA fails to detect the depth aberrations. Finally, we note that the results obtained using the KPCA regularization method outperform the ones obtained by PCA, which seems to over estimate the depth aberrations.

The superiority of the KPCA method is expected since it is able to extract nonlinear structures in the training set of PSFs and is not limited by the vector space assumption discussed in Section 4.3.1. Another noteworthy detail is the repeatability of the proposed BD method to yield consistent results. This can be observed when comparing Fig. 7 and 8, which show that
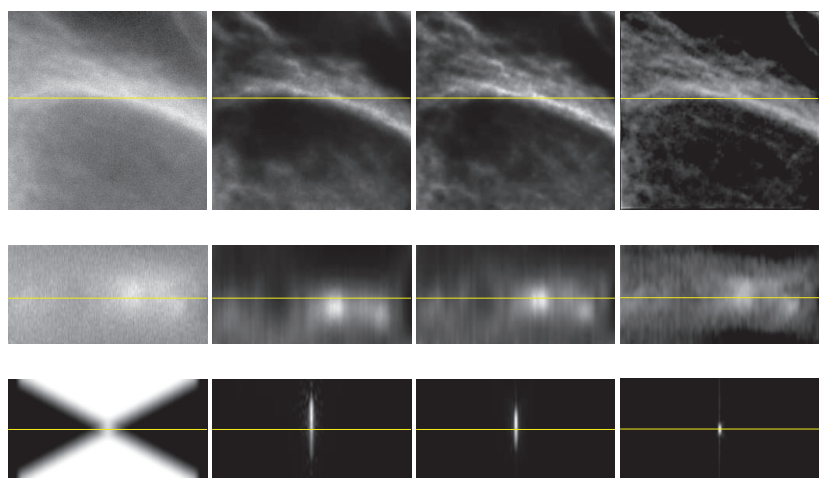

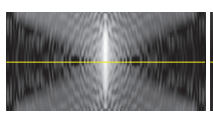

(a)

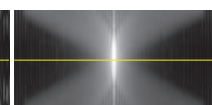

(c)

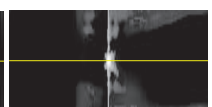

(d)
Fig. 8. Blind deconvolution results for a real depth aberrated image. (a) Original image. (b) Results using PCA regularization. (c) Results using KPCA regularization. (d) Results using AIDA. Top row: $x-y$ image section, second row: $x-z$ image section. PSF initial estimate is displayed in (a). Locators indicate the location of displayed sections. Third row: $r-z$ PSF section, linear scaling. Fourth row: $r-z$ PSF section, logarithmic scaling. Locators indicate $z=0$. 

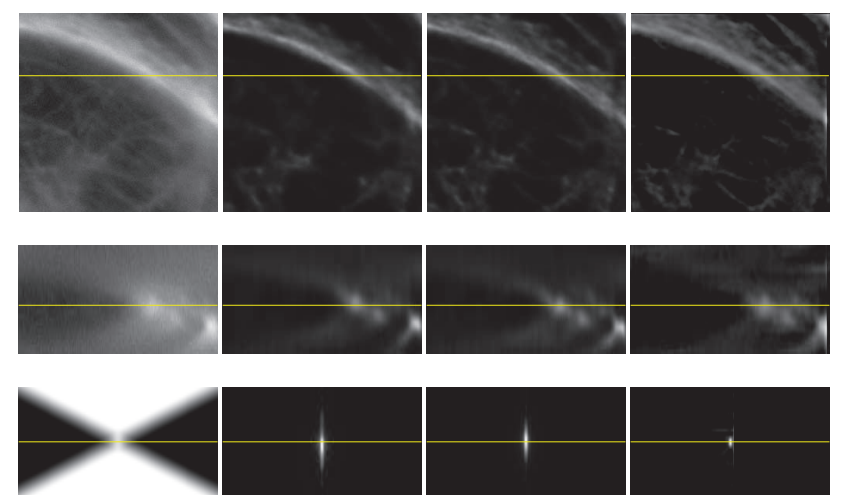


(a) (c)

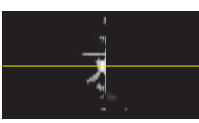

(d)
Fig. 9. Blind deconvolution results for a real nondepth aberrated image. (a) Original image. (b) Results using PCA regularization. (c) Results using KPCA regularization. (d) Results using AIDA. Top row: $x-y$ image section, second row: $x-z$ image section. PSF initial estimate is displayed in (a). Locators indicate the location of displayed sections. Third row: $r-z$ PSF section, linear scaling. Fourth row: $r-z$ PSF section, logarithmic scaling. Locators indicate $z=0$.

similar PSF estimates are obtained when processing different images acquired under similar conditions.

\section{Conclusion and Further Research}

In this work, we presented a new method for blind image deconvolution. We have shown that this method is capable of reconstructing synthetic and real-world acquired WFFM thick specimen images, and producing reconstruction results of high resolution and low noise levels. In addition, the proposed $\mathrm{BD}$ method is capable of reliably providing accurate PSF estimates. This property of the algorithm is demonstrated by overcoming the depth aberration problem in WFFM.

The proposed method compares favorably to a recent BD algorithm [30], especially designed for the reconstruction of 3D microscopic imagery.

The proposed algorithm generalizes a scheme for integrated denoising within the iterative process, which was originally proposed for nonblind deconvolution. We show the potency of this method for BD.

Moreover, we propose a novel method for PSF regularization in $\mathrm{BD}$. This regularization scheme uses machine learning methods which extract the statistical properties of a family of PSFs by example-based learning. This prior is integrated into the iterative process using a simple and efficient formulation and is shown to produce excellent reconstruction results for real world data.

We propose further research directions in related topics. One interesting research path would be exploring other learning methods for the creation of PSF priors. One example for such recent learning method is diffusion maps [9], which is a state-of-the-art method for extracting structures within a given data set. This method has been successfully employed for introducing shape priors for segmentation using active contours [19], [20], and is a natural extension to the method proposed in this work. Another research direction is the application of the proposed algorithm to BD in different fields, other than microscopy, such as astronomical imaging, medical imaging, optics, and photography applications.

\section{ACKNOWLEDGMENTS}

The authors gratefully acknowledge the support of the Israeli Ministry of Science and Technology.

\section{REFEREnCES}

[1] G.R. Ayers and J.C. Dainty, "Iterative Blind Deconvolution Method and Its Applications," Optics Letters, vol. 13, no. 7, pp. 547-549, 1988.

[2] M.J. Booth, M.A.A. Neil, and T. Wilson, "Aberration Correction for Confocal Imaging in Refractive-Index-Mismatched Media," J. Microscopy, vol. 192, no. 2, pp. 90-98, 1998.

[3] M. Born and E. Wolf, Principles of Optics, seventh ed. Cambridge Univ. Press, 1999.

[4] B.E. Boser, I.M. Guyon, and V.N. Vapnik, "A Training Algorithm for Optimal Margin Classifiers," Proc. Fifth Ann. Workshop Computational Learning Theory, pp. 144-152, 1992.

[5] R.W. Boyd, Radiometry and the Detection of Optical Radiation. Wiley, 1983.

[6] P. Campisi and K. Egiazarian, Blind Image Deconvolution: Theory and Applications. CRC Press, 2007.

[7] M. Cannon, "Blind Deconvolution of Spatially Invariant Image Blurs with Phase," IEEE Trans. Acoustics, Speech, and Signal Processing, vol. 24, no. 1, pp. 58-63, Feb. 1976.

[8] A.S. Carasso, "Linear and Nonlinear Image Deblurring: A Documented Study," SIAM J. Numerical Analysis, vol. 36, no. 6, pp. 1659-1689, 1999.

[9] R.R. Coifman, S. Lafon, A.B. Lee, M. Maggioni, B. Nadler, F. Warner, and S.W. Zucker, "Geometric Diffusions as a Tool for Harmonic Analysis and Structure Definition of Data: Diffusion Maps," Proc. Nat'l Academy of Sciences USA, vol. 102, no. 21, pp. 7426-7431, May 2005.

[10] I. Csiszár and G. Tusnády, "Information Geometry and Alternating Minimization Procedures," Statistics and Decisions, Supplement Issue, vol. 1, pp. 205-237, 1984.

[11] S. Dai and Y. Wu, "Motion from Blur," Proc. IEEE Conf. Computer Vision and Pattern Recognition, pp. 1-8, June 2008.

[12] S. Dambreville, Y. Rathi, and A. Tannenbaum, "A Shape-Based Approach to Robust Image Segmentation," Proc. Int'l Conf. Image Analysis and Recognition, vol. 1, pp. 73-183, 2006.

[13] S. Dambreville, Y. Rathi, and A. Tannenbaum, "Shape-Based Approach to Robust Image Segmentation Using Kernel PCA," Proc. IEEE CS Conf. Computer Vision and Pattern Recognition, vol. 1, pp. 977-984, 2006.

[14] S. Dambreville, Y. Rathi, and A. Tannenbaum, "A Framework for Image Segmentation Using Shape Models and Kernel Space Shape Priors," IEEE Trans. Pattern Analysis and Machine Intelligence, vol. 30, no. 8, pp. 1385-1399, Aug. 2008.

[15] D. Datsenko and M. Elad, "Example-Based Single Document Image Super-Resolution: A Global Map Approach with Outlier Rejection," Multidimensional Systems and Signal Processing, vol. 18, pp. 103-121, 2007.

[16] J.B. de Monvel, E. Scarfone, S. Le Calvez, and M. Ulfendahl, "Image-Adaptive Deconvolution for Three-Dimensional Deep Biological Imaging," Biophysical J., vol. 85, no. 6, pp. 3991-4001, 2003.

[17] A.R. De Pierro, "A Modified Expectation Maximization Algorithm for Penalized Likelihood Estimation in Emission Tomography," IEEE Trans. Medical Imaging, vol. 14, no. 1, pp. 132-137, Mar. 1995

[18] N. Dey, L. Blanc-Feraud, C. Zimmer, Z. Kam, J.-C. Olivo-Marin, and J. Zerubia, "A Deconvolution Method for Confocal Microscopy with Total Variation Regularization," Proc. IEEE Int'l Symp. Biomedical Imaging: Nano to Macro, vol. 2, pp. 1223-1226, 2004.

[19] P. Etyngier, F. Segonne, and R. Keriven, "Active-Contour-Based Image Segmentation Using Machine Learning Techniques," Proc. Medical Image Computing and Computer-Assisted Intervention, N. Ayache, S. Ourselin, and A. Maeder, eds., pp. 891-899, 2007. 
[20] P. Etyngier, F. Segonne, and R. Keriven, "Shape Priors Using Manifold Learning Techniques," Proc. IEEE 11th Int'l Conf. Computer Vision, pp. 1-8, 2007.

[21] R. Fergus, B. Singh, A. Hertzmann, S.T. Roweis, and W.T. Freeman, "Removing Camera Shake from a Single Photograph," ACM Trans. Graphics, vol. 25, no. 3, pp. 787-794, 2006.

[22] D.A. Fish, A.M. Brinicombe, E.R. Pike, and J.G. Walker, "Blind Deconvolution by Means of the Richardson-Lucy Algorithm," J. Optical Soc. Am. A, vol. 12, no. 1, pp. 58-65, 1995.

[23] W.T. Freeman, T.R. Jones, and E.C. Pasztor, "Example-Based Super-Resolution," IEEE Computer Graphics and Applications, vol. 22, no. 2, pp. 56-65, Mar./Apr. 2002.

[24] S.F. Gibson and F. Lanni, "Experimental Test of an Analytical Model of Aberration in an Oil-Immersion Objective Lens Used in Three-Dimensional Light Microscopy," J. Optical Soc. Am. A, vol. 9, no. 1, pp. 154-166, Jan. 1992.

[25] J.W. Goodman, Introduction to Fourier Optics, second ed. McGrawHill Companies, Inc., 1996.

[26] P.J. Green, "Bayesian Reconstructions from Emission Tomography Data Using a Modified EM Algorithm," IEEE Trans. Medical Imaging, vol. 9, no. 1, pp. 84-93, Mar. 1990.

[27] M.G.L. Gustafsson, D.A. Agard, and J.W. Sedat, "Sevenfold Improvement of Axial Resolution in 3D Widefield Microscopy Using Two Objective Lenses," Proc. SPIE, pp. 147-156, 1995.

[28] R.M. Haralick and L.G. Shapiro, Computer and Robot Vision. Addison-Wesley Longman Publishing Co., Inc., 1992.

[29] T.J. Holmes, "Blind Deconvolution of Quantum-Limited Incoherent Imagery: Maximum-Likelihood Approach," J. Optical Soc. Am. A, vol. 9, no. 7, pp. 1052-1061, 1992.

[30] E.F.Y. Hom, F. Marchis, T.K. Lee, S. Haase, D.A. Agard, and J.W. Sedat, "AIDA: An Adaptive Image Deconvolution Algorithm with Application to Multi-Frame and Three-Dimensional Data," J. Optical Soc. Am. A, vol. 24, no. 6, pp. 1580-1600, 2007.

[31] J. Jia, "Single Image Motion Deblurring Using Transparency," Proc. IEEE Conf. Computer Vision and Pattern Recognition, pp. 1-8, June 2007.

[32] N. Joshi, R. Szeliski, and D.J. Kriegman, "PSF Estimation Using Sharp Edge Prediction," Proc. IEEE Conf. Computer Vision and Pattern Recognition, pp. 1-8, June 2008.

[33] V. Krishnamurthi, Y.-H. Liu, S. Bhattacharyya, J.N. Turner, and T.J. Holmes, "Blind Deconvolution of Fluorescence Micrographs by Maximum-Likelihood Estimation," Applied Optics, vol. 34, no. 29, pp. 6633-6647, 1995.

[34] D. Kundur and D. Hatzinakos, "Blind Image Deconvolution," IEEE Signal Processing Magazine, vol. 13, no. 3, pp. 43-64, May 1996.

[35] J.-Y. Kwok and I.-H. Tsang, "The Pre-Image Problem in Kernel Methods," IEEE Trans. Neural Networks, vol. 15, no. 6, pp. 15171525, Nov. 2004.

[36] R.L. Lagendijk, J. Biemond, and D.E. Boekee, "Regularized Iterative Image Restoration with Ringing Reduction," IEEE Trans. Acoustics, Speech, and Signal Processing, vol. 36, no. 12, pp. 18741888, Dec. 1988.

[37] R.G. Lane and R.H.T. Bates, "Automatic Multidimensional Deconvolution," J. Optical Soc. Am. A, vol. 4, no. 1, p. 180, 1987.

[38] M.E. Leventon, W.E.L. Grimson, and O. Faugeras, "Statistical Shape Influence in Geodesic Active Contours," Proc. IEEE Conf. Computer Vision and Pattern Recognition, vol. 1, pp. 316-323, 2000.

[39] D. Li, R.M. Mersereau, and S. Simske, "Blind Image Deconvolution through Support Vector Regression," IEEE Trans. Neural Networks, vol. 18, no. 3, pp. 931-935, May 2007.

[40] D. Li, R.M. Mersereau, and S. Simske, "Blur Identification Based on Kurtosis Minimization," Proc. IEEE Int'l Conf. Image Processing, vol. 1, pp. I-905-8, Sept. 2005.

[41] L. Lucy, "An Iterative Technique for the Rectification of Observed Distributions," The Astronomical J., vol. 79, pp. 745-754, 1974.

[42] F. Macias-Garza, A.C. Bovik, K.R. Diller, S.J. Aggarwal, and J.K. Aggarwal, "Digital Reconstruction of Three-Dimensional Serially Sectioned Optical Images," IEEE Trans. Acoustics, Speech, and Signal Processing, vol. 36, no. 7, pp. 1067-1075, July 1988.

[43] J. Markham and J.-A. Conchello, "Parametric Blind Deconvolution of Microscopic Images: Further Results," Proc. SPIE ThreeDimensional and Multidimensional Microscopy: Image Acquisition and Processing V, C.J. Cogswell, J.-A. Conchello, T. Wilson, T.T. Lu, and J.M. Lerner, eds., pp. 38-49, Jun. 1998.
[44] S. Mika, B. Schölkopf, A.J. Smola, K.-R. Müller, M. Scholz, and G. Rätsch, "Kernel PCA and De-Noising in Feature Spaces," Advances in Neural Information Processing Systems 11, M.S. Kearns, S.A. Solla, and D.A. Cohn, eds., MIT Press, 1999.

[45] R. Nakagaki and A.K. Katsaggelos, "A VQ-Based Blind Image Restoration Algorithm," IEEE Trans. Image Processing, vol. 12, no. 9, pp. 1044-1053, Sept. 2003.

[46] A.V. Oppenheim and J.S. Lim, "The Importance of Phase in Signals," Proc. IEEE, vol. 69, no. 5, pp. 529-541, May 1981.

[47] K. Panchapakesan, D.G. Sheppard, M.W. Marcellin, and B.R. Hunt, "Blur Identification from Vector Quantizer Encoder Distortion," IEEE Trans. Image Processing, vol. 10, no. 3, pp. 465-470, Mar. 2001.

[48] P. Pankajakshan, B. Zhang, L. Blanc-Feraud, Z. Kam, J.-C. OlivoMarin, and J. Zerubia, "Blind Deconvolution for DiffractionLimited Fluorescence Microscopy," Proc. Fifth IEEE Int'l Symp. Biomedical Imaging: From Nano to Macro, pp. 740-743, 2008.

[49] K. Pearson, "On Lines and Planes of Closest Fit to Systems of Points in Space," Philosophical Magazine, vol. 2, no. 6, pp. 559-572, 1901.

[50] R. Raskar, A. Agrawal, and J. Tumblin, "Coded Exposure Photography: Motion Deblurring Using Fluttered Shutter," ACM Trans. Graphics, vol. 25, no. 3, pp. 795-804, 2006.

[51] Y. Rathi, S. Dambreville, and A. Tannenbaum, "Statistical Shape Analysis Using Kernel PCA," Proc. SPIE, Electronic Imaging, 2006.

[52] S.J. Reeves and R.M. Mersereau, "Blur Identification by the Method of Generalized Cross-Validation," IEEE Trans. Image Processing, vol. 1, no. 3, pp. 301-311, July 1992.

[53] W.H. Richardson, "Bayesian-Based Iterative Method of Image Restoration," J. Optical Soc. Am. A, vol. 62, no. 1, pp. 55-59, 1972.

[54] M. Rousson and N. Paragios, "Shape Priors for Level Set Representations," Proc. Seventh European Conf. Computer VisionPart II, pp. 78-92, 2002.

[55] L.I. Rudin, S. Osher, and E. Fatemi, "Nonlinear Total Variation Based Noise Removal Algorithms," Physica D, vol. 60, nos. 1-4, pp. 259-268, 1992.

[56] B.A. Scalettar, J.R. Swedlow, J.W. Sedat, and D.A. Agard, "Dispersion, Aberration and Deconvolution in Multi-Wavelength Fluorescence Images," J. Microscopy, vol. 182, no. 1, pp. 50-60, Apr. 1996.

[57] B. Schölkopf, A. Smola, and K.-R. Müller, “Nonlinear Component Analysis as a Kernel Eigenvalue Problem," Neural Computation, vol. 10, no. 5, pp. 1299-1319, 1998.

[58] Q. Shan, J. Jia, and A. Agarwala, "High-Quality Motion Deblurring from a Single Image," ACM Trans. Graphics, vol. 27, no. 3, pp. 1-10, 2008.

[59] L.A. Shepp and Y. Vardi, "Maximum Likelihood Reconstruction for Emission Tomography," IEEE Trans. Medical Imaging, vol. 1, no. 2, pp. 113-122, Oct. 1982.

[60] J.-L. Starck, E. Pantin, and F. Murtagh, "Deconvolution in Astronomy: A Review," Publications of the Astronomical Soc. of the Pacific, vol. 114, pp. 1051-1069, 2002.

[61] J. Stockham, T.G., T.M. Cannon, and R.B. Ingebretsen, "Blind Deconvolution through Digital Signal Processing," Proc. IEEE, vol. 63, no. 4, pp. 678-692, Apr. 1975.

[62] P.A. Stokseth, "Properties of a Defocused Optical System," J. Optical Soc. Am. A, vol. 59, no. 10, pp. 1314-1321, 1969.

[63] N. Streibl, "Three-Dimensional Imaging by a Microscope," J. Optical Soc. Am. A, vol. 2, no. 2, pp. 121-127, 1985.

[64] A. Tsai, J. Yezzi, A.W. Wells, C. Tempany, D. Tucker, A. Fan, W. Grimson, and A. Willsky, "A Shape-Based Approach to the Segmentation of Medical Imagery Using Level Sets," IEEE Trans. Medical Imaging, vol. 22, no. 2, pp. 137-154, Feb. 2003

[65] F. Tsumuraya, N. Miura, and N. Baba, "Iterative Blind Deconvolution Method Using Lucy's Algorithm," Astronomy and Astrophysics, vol. 282, pp. 699-708, Feb. 1994.

[66] Y. Yang, N.P. Galatsanos, and H. Stark, "Projection-Based Blind Deconvolution," J. Optical Soc. Am. A, vol. 11, no. 9, pp. 2401-2409, 1994.

[67] Y.-L. You and M. Kaveh, "Blind Image Restoration by Anisotropic Regularization," IEEE Trans. Image Processing, vol. 8, no. 3, pp. 396407, Mar. 1999.

[68] L. Yuan, J. Sun, L. Quan, and H.-Y. Shum, "Image Deblurring with Blurred/Noisy Image Pairs," ACM Trans. Graphics, vol. 26, no. 3, pp. 1-10, 2007. 
[69] B. Zhang, J. Zerubia, and J.-C. Olivo-Marin, "Gaussian Approximations of Fluorescence Microscope Point-Spread Function Models," Applied Optics, vol. 46, no. 10, pp. 1819-1829, 2007.

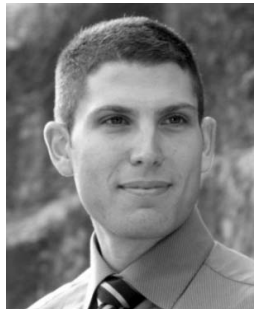

Tal Kenig received the BSc degree from the Biomedical Engineering Faculty at the Technion in 2005 and the MSc degree from the Faculty of Electrical Engineering at the Technion in 2009. From 2004 to 2007, he was with General Electric Healthcare, Functional Imaging, working on the development of medical image processing and analysis algorithms. He is currently with Medic Vision Imaging Solutions, where he leads the algorithm development team, in the field of medical imaging. His research interests include medical and biological image processing and analysis, computer vision, inverse problems, and machine learning.



Zvi Kam received the $\mathrm{PhD}$ degree in solid state physics in 1971 from the Technion, Israel Institute of Technology. He continued his postdoctoral research in biophysics at the University of California San Diego, and in 1975 he joined the Weizmann Institute of Science, Rehovot, Israel, where he is a professor of biophysics in the Department of Molecular Cell Biology. He is applying light microscopy to cell biology. Recent work is focused on cell-based screens as a way to probe complex multimolecular mechanisms. To better relate microscope imaging to cellular functions, a high-resolution automated screening microscope was built and associated with quantitative image analysis pipeline for high-throughput analysis.

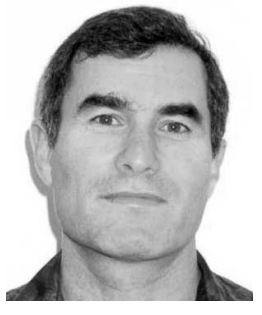

Arie Feuer received the $\mathrm{BSc}$ and $\mathrm{MSc}$ degrees from the Technion in 1967 and 1973, respectively, and the $\mathrm{PhD}$ degree from the Yale University in 1978. He has been with the Electrical Engineering Department at the Technion-Israel Institute of Technology since 1983, where he is currently a chaired professor and head of the Control and Robotics Lab. From 1967 to 1970 , he was in industry working on automation design and, between 1978 and 1983, with Bell Labs in Holmdel, New Jersey. Between the years 1994 and 2002, he served as the president of the Israel Association of Automatic Control and was a member of the IFAC Council during the years 2002-2005. He is a fellow of the IEEE. In the last 20 years, he has been regularly visiting the Electrical Engineering and Computer Sciende Department at the University of Newcastle. His current research interests include resolution enhancement of digital images and videos, 3D video and multiview data, sampling and combined representations of signals and images, and adaptive systems in signal processing and control.

For more information on this or any other computing topic, please visit our Digital Library at www.computer.org/publications/dlib. 